\title{
Photodissociation of molecules: the microscopic path of a molecular decay
}

\author{
J. Robert Huber
}

Physikalisch-Chemisches Institut der Universität Zürich, Winterthurerstr. 190, CH-8057 Zürich, Switzerland

\begin{abstract}
The state-selective photodissociation of methylnitrite
$\mathrm{CH}_{3} \mathrm{ONO}\left(\mathrm{SO}_{\mathrm{O}}, \mathrm{v}\right)+\mathrm{h \nu} \rightarrow \mathrm{CH}_{3} \mathrm{O} \cdot+\mathrm{NO}(t, r, v)$
\end{abstract}

is reviewed and the dynamics of the bond-breaking process is analysed in connection with an ab initio $s_{1}$-potential energy surface of the parent molecule. Experimental and theoretical results reveal an interesting picture of the dissociation path of a polyatomic molecule which decays by vibrational predissociation and a strong exit channel interaction.

\section{INTRODUCTION}

If a polyatomic molecule absorbs light with an energy exceeding the dissociation energy of its weakest bond, fragmentation can occur which produces products in excited states. The dynamics of the process, which in turn is given by the potential energy surface of the excited parent molecule, determines the fragmentation pathway and energy distribution in the photoproducts (ref. 1). According to the dissociation dynamics we may distinguish between a direct dissociation and a predissociation. In the case of a direct dissociation the excited state potential energy surface (PES) is antibonding and strongly repulsive along one or more coordinates yielding an extremly fast (< 10 fs) bond-breaking process ( $\mathrm{ff}$. Fig. 1a). The absorption spectrum of the parent molecule is, therefore, broad and structureless. Predissociation, on the other hand, may be the result of an excited state PES characterized by a local well along the reaction coordinate as depicted in Fig. Ib.

The potential well can support quasi-bound levels of the excited molecule and due to the barrier, the decay of the excited molecule is slow compared to direct dissociation ( $100 \mathrm{fs})$. The decay is induced either by tunneling through the barrier in the case of an adiabatic process, and/or by coupling between internal and dissociative modes in the case of a nonadiabatic process. The potential well acts like a short-term waiting room for the excited
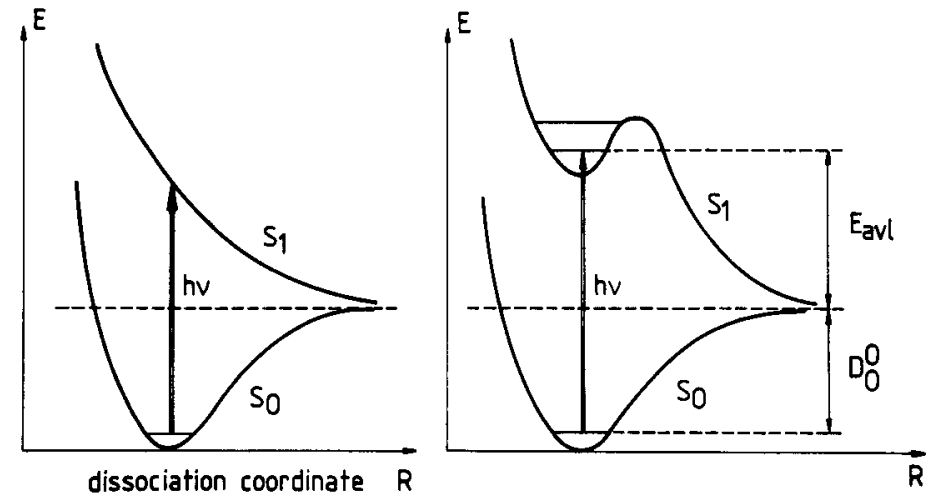

Fig. 1. Direct dissociation, left (a) and indirect dissociation (predissociation), right (b) 
molecule to decay and it gives rise to pronouced structures in the absorption spectrum which reflect the energy spacings of the quasi-bound states supported by the potential well. Under these conditions the excitation process and the decay of the excited complex can be treated separately (see below).

In the present communication we will focus on predissociation, in particular, on the experimental and theoretical results obtained from a photodissociation study of methyinitrite excited into different vibrational levels of the electronic state $S_{1}$ (refs. 2 - 11). It is shown that methylnitrite is an example of vibrational predissociation and an excellent system to explore in detail the microscopic decay path of an excited polyatomic molecule.

\section{POTENTIAL ENERGY SURFACE}

The weak $S_{I}\left(n x^{*}\right) \leftarrow S_{0}$ transition of methylnitrite is localized in the $0-N=0$ moiety. Figure 2 shows the spectrum which is dominated by the absorption of the cis-conformer of $\mathrm{CH}_{3}$ ONO. It is characterized by a well defined progression of the $N=0$ stretching vibration $v_{3} \sim 1000 \mathrm{~cm}^{-1}$. Excitation into a vibronic level assoclated with $v_{3}\left(s_{1}, n^{*}\right)$ results in prompt dissociation producing NO and a methoxy radical (refs. $2-7$ ).

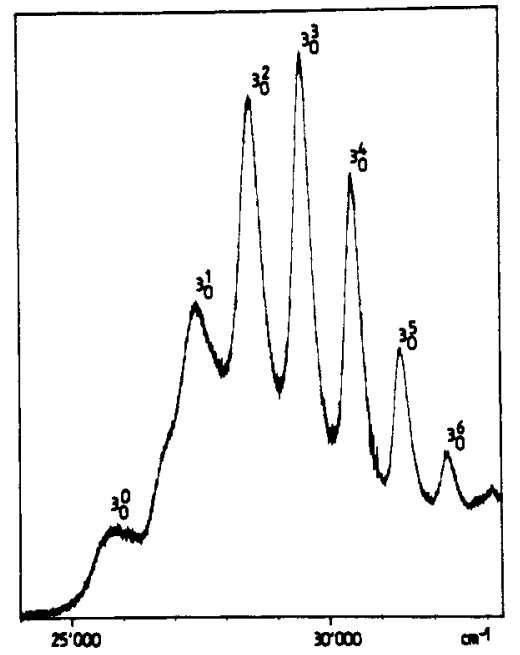

Fig. 2. $s_{1} \leftarrow s_{0}$ absorption spectrum of $\mathrm{CH}_{3}$ ONO in the gas phase at room temperature. The $v_{3}$ progression is due to the $N=0$ stretching vibration. The spectrum is dominated by the cis-form.

Owing to the localized nature of the $n \pi^{*}$ excitation, it was expected that a two-dimensional PES involving the coordinates $r_{N}=0$ and $R_{O-N}$ could provide a realistic description for the dissociation process. The result of an ab initio (MCSCF) calculation (ref. 5) of such a two-dimensional $S_{1}$-PES for cis- $\mathrm{CH}_{3} \mathrm{ONO}$ is given in Fig. 3. Two features of this PES are of importance. First, a shallow well is evident along the dissociation coordinate $R_{O-N}$ and close to the Franck-Condon region (excitation region) probably caused by an avoided crossing with a higher state. If this well can support quasi-bound energy levels, we encounter a situation very similar to the one described in Fig. 1b. Second, the minimum energy dissociation path is strongly curved which means that the potential parameters of the $\mathrm{N}=0$ group, namely the bond length and the force constant, depend significantly on the $\mathrm{CH}_{3} \mathrm{O}--\mathrm{NO}$ separation. This fact indicates a strong vibrational-translational coupling that is responsible for the redistribution of internal energy during the separation of the fragments and thus to a great extent to the final energy partitioning (ref. 10). A very similar picture was found for the related molecule HONO (refs. 12, 13).

Based on these findings the photodissoclation can be treated as a two-step process (ref. 10)

$$
\begin{array}{ll}
\mathrm{CH}_{3} \mathrm{ONO}\left(\mathrm{s}_{\mathrm{O}} \mid \mathrm{n}=\mathrm{O}\right)+\mathrm{hv} & +\mathrm{CH}_{3} \mathrm{ONO}\left(\mathrm{s}_{1} \mid \mathrm{n}^{*}\right) \\
\mathrm{CH}_{3} \mathrm{ONO}\left(\mathrm{s}_{1} \mid n^{*}\right) & +\mathrm{NO}(t, v, r, e)+\mathrm{CH}_{3} \mathrm{O}(t, v, r)
\end{array}
$$

where $n$ and $n *$ denote the (effective) quantum numbers of $v_{3}$ in the $s_{0}$ and $s_{1}$ states, respectively. 


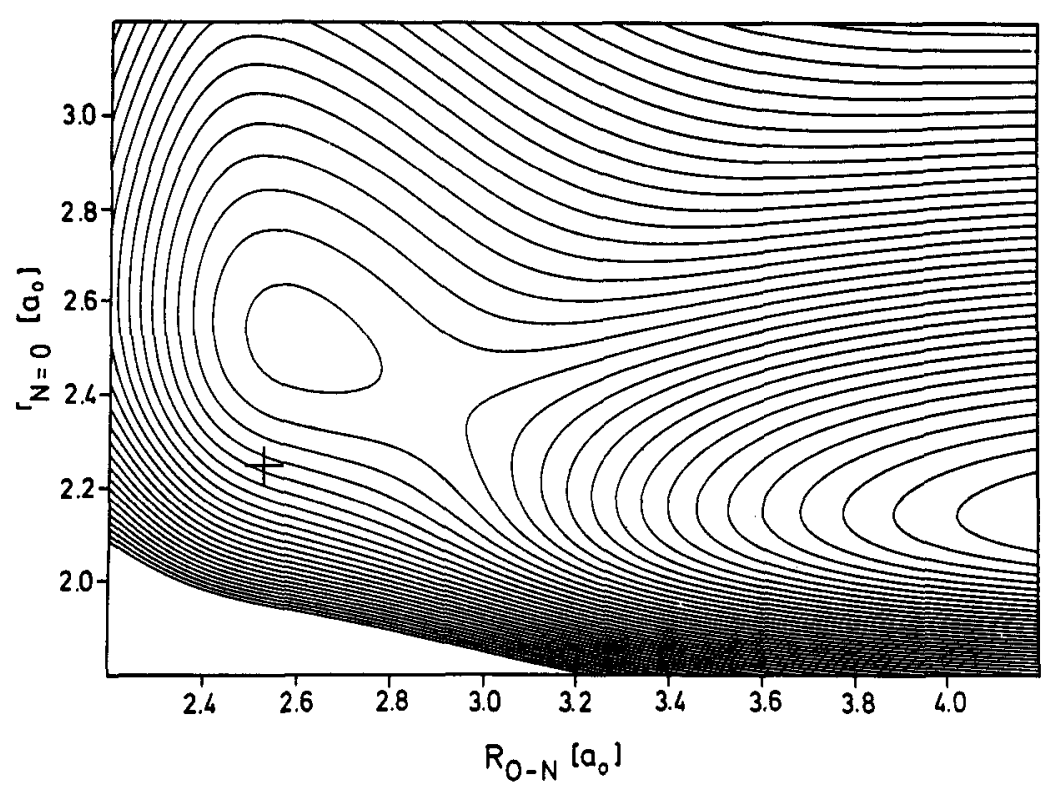

Fig. 3. Contour plot of the ab initio $\mathrm{s}_{1}$-potential surface of $\mathrm{CH}_{3} \mathrm{ONO}$ (ref. 5). The energy spacing is $0.136 \mathrm{eV}$. The cross indicates the equilibrium of $\mathrm{S}_{0}$ (Franck-Condon region).

\section{EXPERIMENTAL RESULTS}

The photodissociation of $\mathrm{CH}_{3}$ ONO was investigated after excitation into the $30^{1}(363.9 \mathrm{~nm}), 30^{2}(350.8 \mathrm{~nm})$ and $30^{3}(338.4 \mathrm{~nm})$ vibronic bands (cf. Fig. 2). The energy $E_{a v l}$ which is available to be distributed among the internal degrees of freedom and the recoil of the NO and $\mathrm{CH}_{3} \mathrm{O}$ fragments is given by

$$
E_{\text {avI }}=E_{0}\left(\mathrm{CH}_{3} \mathrm{ONO}\right)+\mathrm{h \nu}-\mathrm{D}_{0} \mathrm{O}
$$

where $\mathrm{E}_{\mathrm{O}}\left(\mathrm{CH}_{3} \mathrm{ONO}\right) \sim 700 \mathrm{~cm}^{-1}$ is the internal energy of the parent, hv the excitation energy, and $D_{0}{ }^{O}=14500 \pm 400 \mathrm{~cm}^{-1}$ the dissociation energy of $\mathrm{CH}_{3} \mathrm{O}-\mathrm{NO}$. For the three excitation wavelengths used we obtain $\mathrm{E}_{\text {avl }}=13750$, 14730 and $15770 \mathrm{~cm}^{-1}$, respectively (refs. 3,9 ).

We probed the internal energy distribution and the translational energy of the No fragments emerging from the dissociation using an apparatus and methods described elsewhere (ref. 14). Briefly, two polarized laser beams, one to dissociate $\mathrm{CH}_{3} \mathrm{ONO}$ and one to probe NO, were crossed in a gas cell flowed continuously with $\mathrm{CH}_{3}$ ONO at a pressure of $\sim 100 \mathrm{mTorr}$. The probe laser pulse was opticaliy delayed by $10 \mathrm{~ns}$ relative to the dissociation laser pulse. In order to protect the parent molecule from the analyzing laser light, nascent $\mathrm{NO}\left(\mathrm{X}^{2} \Pi\right)$ was probed using two photon laser induced fluorescence (LIF) ( $2 \mathrm{X} \sim$ $450 \mathrm{~nm}$ ). Figure 4 shows the rotational spectrum of a single vibrational level of nascent NO taken $10 \mathrm{~ns}$ after the photodissociation of $\mathrm{CH}_{3}$ ONO. Such a spectrum measured under different polarization conditions for all populated

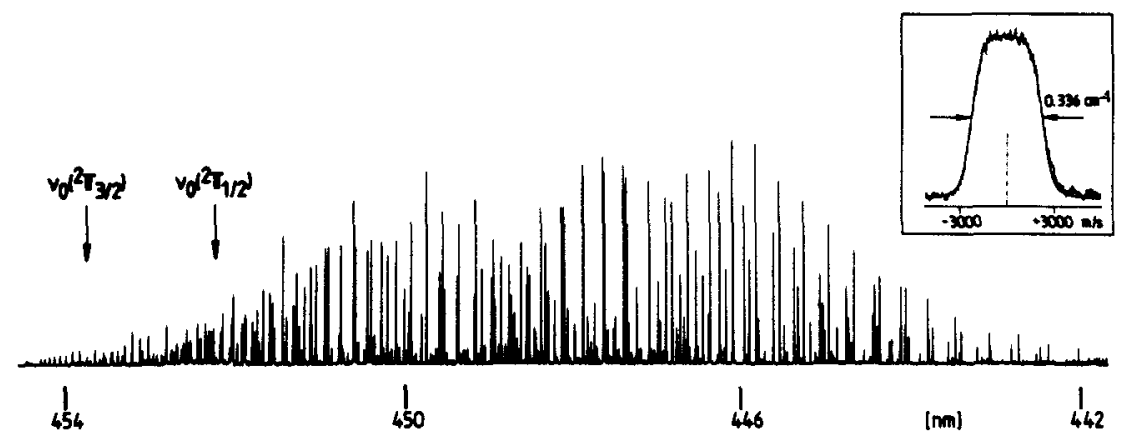

Fig. 4. Rotational (LIF) spectrum of nascent No( $v^{\prime \prime}=0$ ) after photodissoclation of $\mathrm{CH}_{3} \mathrm{ONO}$ at $363.9 \mathrm{~mm}$. The insert shows the Doppler profile of a selected rotational ilne. 


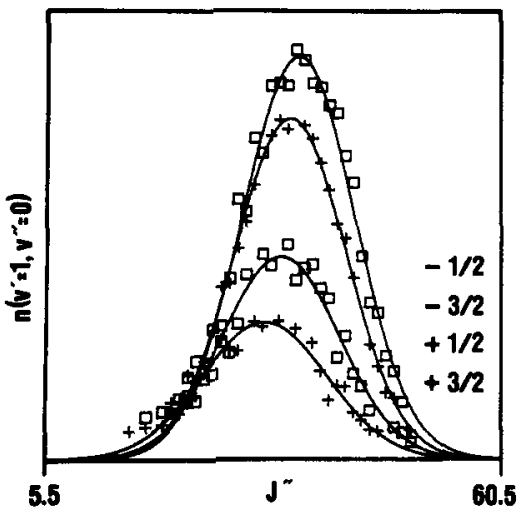

Fig. 5. Rotational state population distributions of the four sublevels of NO $\left(v^{\prime \prime}=0\right)$ created in the photodissociation of $\mathrm{CH}_{3}$ ONO $\left(\mathrm{S}_{1}, \mathrm{n}=1\right)$.
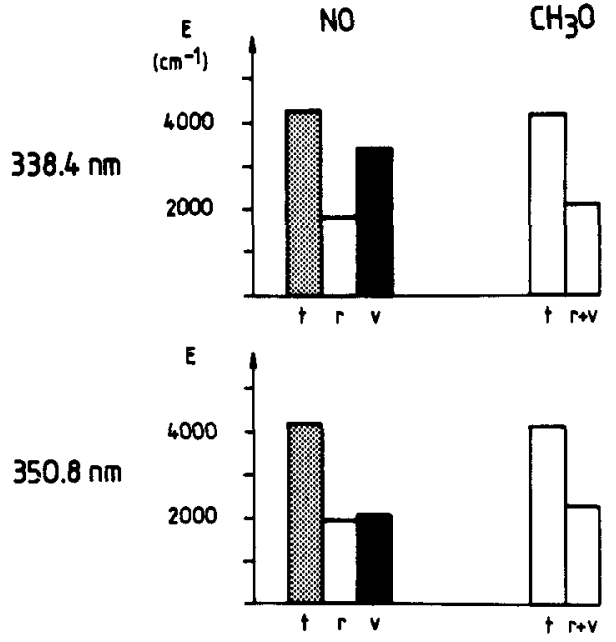

$363.9 \mathrm{~nm}$

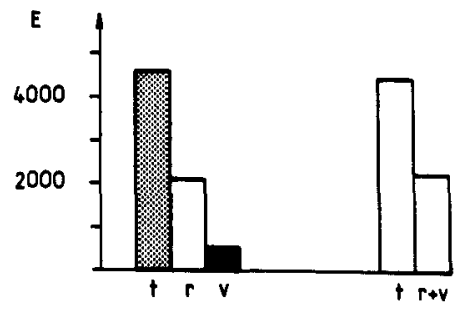

Fig. 6. Energy partitioning ( $t=t r a n s-$ lational, v=vibrational, r=rotational) into the NO and $\mathrm{CH}_{3} \mathrm{O}$ fragments after photodissociation of $\mathrm{CH}_{3} \mathrm{ONO}$ at three different excitation wavelengths $\left(30^{1}, 30^{2}, 30^{3}\right)$.

vibrational states of No contains the basic information of the internal state distribution. From the intensities one obtains the state populations and from the intensity variation for different probe laser polarizations, the rotational alignment and hence, information about the geometry of the dissociation process (ref. 15). Figure 5 shows the rotational state distribution of nascent NO in its zeroth vibrational level. In the case of the No fragment, the spectrum can be disentangled into 4 rotational sub-spectra belonging to the two spin-levels $1 / 2$ and $3 / 2$ and their two $\Lambda$-sublevels $\left(\pi_{ \pm 1 / 2}\right.$, $\left.I_{+3} / 2\right)$. These spectra provide further information about the energetics and the geometry of the bond-breaking process (ref. 16).

Finally, the recoll velocity distribution and the translational energy of the fragment are deduced from Doppler profile measurements shown as inserts in Fig. 4. Furthermore, the Doppler profile analysis yields the parameter $\beta$ which is a measure of the spatial anisotropy of the fragment (ref. 17). Since the degree of this anisotropy depends on the dissociation time tdiss and the angular frequency of rotation of the excited parent molecule, the

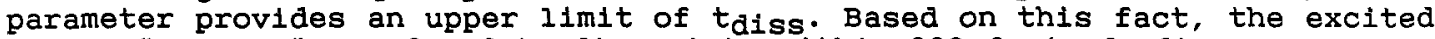
$\mathrm{CH}_{3}$ ONO "complex" was found to dissociate within 200 fs (ref. 6).

Having determined the complete state distribution and the three-dimensional recoil velocity distribution of NO, we also know the translational energy of $\mathrm{CH}_{3} \mathrm{O}$ through linear momentum conservation and the sum of its rotational and vibrational energy content through energy conservation.

Figure 6 shows the energy partitioning of $E_{a v l}$ into the translational, vibrational and rotational degrees of freedom for the two fragments after excitation of $\mathrm{CH}_{3}$ ONO into the $\mathrm{N}=0$ stretching overtones $30^{1}, 3_{0}{ }^{2}$ and $30^{3}$ of the $S_{1}$ state (ref. 9). The electronic excitation of No due to population of the higher spin-state $\pi_{3 / 2}\left(\sim 120 \mathrm{~cm}^{-1}\right.$ above $\left.I_{1 / 2}\right)$ amounts to about $50 \mathrm{~cm}^{-1}$ and is not included. 


\section{DISCUSSION}

We first consider the geometrical properties of the dissociation process (see eqs. $1-2$ and FIg. 7). Methylnitrite is a planar molecule with respect to its C-O-NO frame in the $s_{0}$ and $s_{1}$ state. The $s_{1} \leftarrow s_{0}$ transition dipole moment $\vec{\mu}$ is perpendicular to this plane. The photodissociation creates No fragments with rotational populations preferentialiy in large |M| values implying the angular momentum vector $\vec{J}$ to be parallel to $\vec{\mu}$. This fact indicates a dissociation process which proceeds preferentially in the plane of the molecule. The same conclusion is also obtained by an analysis of the $\Lambda$-sublevel populations and the vector correlation between $J$ and the fragment recoil velocity vector $\vec{v}$ (ref. 6 ).

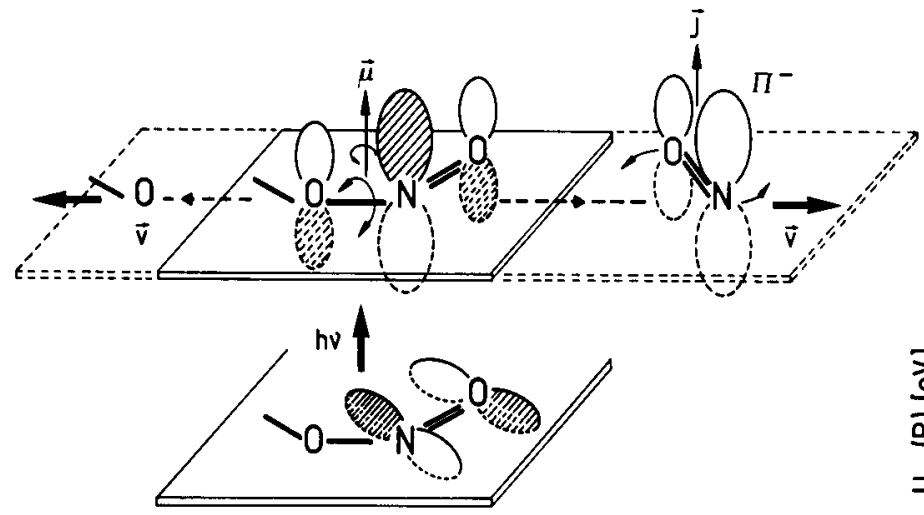

Fig. 7. Geometry of the dissociation process which takes place within $200 \mathrm{fs}$.

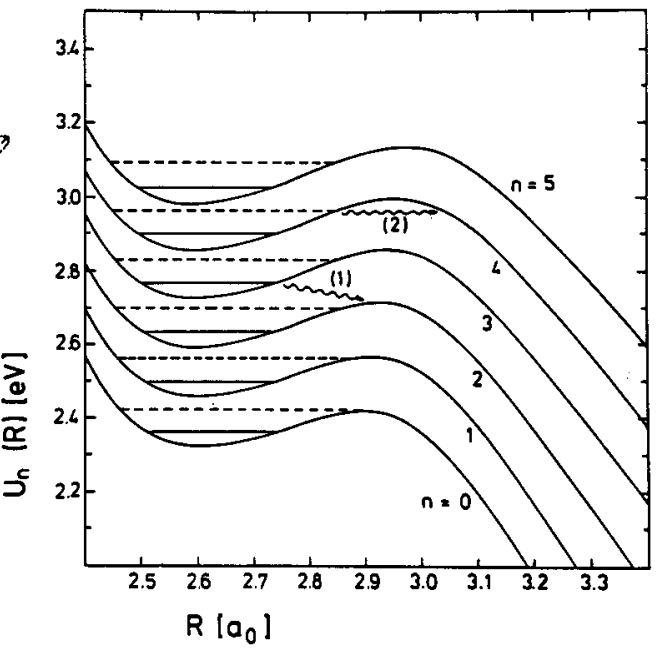

Fig. 8. Adiabatic potentials $U\left(R_{O-N}\right)$ in the region of the local potential well (cf. Fig. 3). The solid and dashed horizontal lines indicate the resonance energies (ref. 10). The arrows indicate the two decay mechanisms, i.e. vibrational predissociation (1) and tunneling (2).

A more subtle picture of the dissociation mechanism is revealed by the $s_{1}$ potential surface (refs. 5, 10) reproduced in Fig. 3. After absorption of a photon the system is prepared in the Franck-Condon region and starts to evolve in time. Owing to the shallow well in the PES, an immediate dissociation is prohibited and the activated species is caught for a few vibrational periods ( $t_{N=0} \sim 30$ fs) in the potential well. In a second step the decay of the excited molecule is induced by a nonadiabatic and/or a tunneling process. In the first case vibrational-translational coupling causes one quantum of the $N=0$ vibration to be transfered to the dissociation mode.

This situation is illustrated in Fig. 8 which exhibits a series of vibronic PES for $n^{*}=0,1,2,3,4,5$. From the $n^{\star}$ PES the decaying species passes to the $n{ }^{*}-1$ PES and dissociates along this potential producing No fragments preferentially in the $n^{*}-1$ vibrational level. Depending on the exit channel interaction further deactivations to $n^{*}-2$ or $n^{*}-3$ are also feasible. The tunneling mechanism, on the other hand, provides an adiabatic dissociation pathway along the initial potential curve and gives rise to No products mainly in $\mathrm{n}^{*}$. The photodissociation of $\mathrm{CH}_{3}$ ONO is thus a nice example of vibrational predissociation which involves the special feature of a strong exit channel interaction. The final state distribution in the fragments is mainly determined in the exit channel. Even though the activated species performs several vibrations during its lifetime in the potential well, the fragment state distribution is highly non-statistical. A similar behaviour was also found for the related molecule HONO (ref. 13). 
The energetics of the dissociation process is summarized in Fig. 6 . The reader is refered to ref. 9 for the detalls of the energy partitioning. 27 33 of the available energy goes into the translational motion of the No fragment and 12 - 15 into its rotational degree of freedom. While translation and rotation are only slightly dependent on the overtone excitation, the vibrational energy content of No is strongly influenced. If the overtone excitation $\left(v_{3}{ }^{n}\right)$ of the parent molecule is increased, the additional vibrational energy is channeled exclusively into the No vibration of the fragment (ref. 3). This high selectivity of vibrational excitation is a consequence of the vibrational predissociation process of $\mathrm{CH}_{3} \mathrm{ONO}$ described above.

Recently we have extended our ab initio calculation of the $\mathrm{s}_{1}-\mathrm{PES}$ of $\mathrm{CH}_{3} \mathrm{ONO}$ to include a third dimension. Beside $r_{N=0}$ and $R_{O-N}$ we also varied the angle $O-N=0$. Based on these PES we also performed classical trajectory calculations to obtain the rotational state distribution in No. The results of these calculations agree well with the experimental findings and thus complete the analysis of the dynamics and state distributions in the $s_{1}$ dissociation of $\mathrm{CH}_{3} \mathrm{ONO}$.

Although the photodissociation of methylnitrite investigated by the LIF technique produces a wealth of data to explore the decay of a polyatomic molecule, a convincing interpretation of the data requires -as in generala potential energy surface of rather good quality (ref. 18). Such a PES forms the basis for quantum mechanical studies and classical trajectory calculations, which in turn yield the photodissociation dynamics and the state distributions of the products. Fortunately $\mathrm{CH}_{3}$ ONO turned out to be a favorable molecule with respect to this requirement, and the result provided a deep insight into the dynamics of a photodissociation process in a polyatom1c molecule, in particular, vibrational predissociation in an excited electronic state.

\section{Acknowledgement}

Support of this work by the Schweizerischer Nationalfonds is gratefully acknowledged. I am indebted to Drs. Martin Dubs, Ueli Bruhlmann, Marco Nonella, Reinhard Schinke (Gottingen) and Peter Felder whose contributions made this work possible. I also thank Dr. Michael P. Docker for critically reading the manuscript.

\section{REFERENCES}

1. H. Okabe, Photochemistry of Small Molecules, Wiley, New York (1978),

2. F. Lahman1, C. Lardeux and D. Solgadi, Chem. Phys. Lett. 102, 523-528 (1983).

3. O. Benoist d'Azy, F. Lahmani, C. Lardeux and D. Solgadi, Chem. Phys. 94, 247-256 (1985).

4. F. Lahmani, C. Lardeux and D. Solgadi, Chem. Phys. Lett. 129, 24-30 (1986).

5. M. Nonella and J.R. Huber, Chem. Phys. Lett. 131, 376-379 (1986).

6. U. Brühlmann, M. Dubs and J.R. Huber, J. Chem. Phys. 86, 1249-1257 (1987).

7. B.A. Keller, P. Felder and J.R. Huber, J. Phys. Chem. 91, 1114-1120 (1987).

8. P. Felder, B.A. Keller and J.R. Huber, Z. Phys. D 6, 185-192 (1987).

9. U. Brühlmann and J.R. Huber, Z. Phys. D $7,1-8(19 \overline{8} 7)$.

10. S. Hennig, V. Engel, R. Schinke, M. Noneila and J.R. Huber, J. Chem. Phys. 87, 3522-3529 (1987).

11. U. Bruhlmann and J.R. Huber, Chem. Phys. Lett. 143, 199-203 (1987).

12. R. Vasudev, R.N. Zare and R.N. Dixon, J. Chem. Phys. 80, 4863-4878 (1984).

13. S. Hennig, A. Untch, R. Schinke, M. Nonella and J.R. Huber, Chem. Phys., submitted

14. M. Dubs, U. Brühlmann and J.R. Huber, J. Chem. Phys. 84, 3106-3119 (1986).

15. C.H. Greene and R.N. Zare, Annu. Rev. Phys. Chem. 33, 119-150 (1982).

16. P. Andresen and E.W. Rothe, J. Chem. Phys. 82, 3634-3640 (1985).

17. R.N. Zare, Mol. Photochem. 4, 1-37 (1972).

18. R. Schinke, V. Engel, S. Heñnig, K. Weide and A. Untch, Ber. Bunsenges. f. Phys. Chem. (1988) in press 